\title{
Analysis of the Effect of Nonlinear Low Noise Amplifier with Memory for Wideband Spectrum Sensing
}

\author{
Attaphongse Taparugssanagorn*, Kenta Umebayashi ${ }^{\dagger}$, Janne Lehtomäki $^{\ddagger}$, and Carlos Pomalaza-Ráez ${ }^{\S}$ \\ ${ }^{*}$ Wireless Information Security and Eco-Electronics Research Unit, National Electronics and Computer Technology Center, \\ Pathum Thani, Thailand, Email: attaphongse.taparugssanagorn@ nectec.or.th \\ ${ }^{\dagger}$ Tokyo University of Agriculture and Technology, Tokyo, Japan \\ ${ }^{\ddagger}$ Centre for Wireless Communications, University of Oulu, Finland
}

$\S$ College of Engineering, Technology, and Computer Science, Department of Engineering, Purdue University, Indiana, USA

\begin{abstract}
In wideband sensing receivers, especially in lowcost mass-product devices, the sensing operation is usually done in a range where the RF front-end components, such as the LNAs exhibit a nonlinear behavior. Consequently, intermodulation (IMD) and crossmodulation (XM) are generated and cause distortion in the spectrum sensing region. This paper investigates the impact on the threshold level setting of a wideband energy detection based spectrum sensing caused by a nonlinear LNA at the radio frequency ( $R F)$ front-end of a wideband cognitive radio receiver. The third and fifth order terms of the expansion of the nonlinearity characteristics are modeled using a memorypolynomial model. The main contribution of this study is the derivation of a proper threshold level for different frequency bins.
\end{abstract}

Index Terms-Wideband spectrum sensing, nonlinear distortion, memory-polynomial, exponential distribution.

\section{INTRODUCTION}

Cognitive radio $(\mathrm{CR})$ is an advanced software-defined radio that can be programmed and configured dynamically [1]. It can efficiently use the wireless channels in its vicinity. Such a radio automatically detects available channels in the wireless spectrum, then accordingly changes its transmission or reception parameters to allow more concurrent wireless communications in a given spectrum band at one location. One of the main functions of cognitive radios is spectrum sensing, i.e., detecting unused spectrum and sharing it, without harmful interference to other users [2,3]. This kind of opportunistic spectrum access introduces the possibility for a secondary user, who does not have a license for a particular band, to transmit when the primary is not using that band. Therefore, the secondary user should search for spectrum bands left unused by several primary users, and operate over such spaces.

There has been extensive work on spectrum sensing in a narrowband setup. Narrowband spectrum sensing consists of observing a relatively narrow radio spectrum band and deciding whether there is a signal or there is noise [4]. When a wideband spectrum is assigned to a number of primary users, secondary users can search for unoccupied channels (spectrum holes) within the wideband spectrum and communicate in that band. The traditional way for detecting holes in a wideband spectrum is channel-by-channel scanning [5-8]. In order to implement this method, an RF front-end with a bank of tunable and narrow bandpass filters is needed. The occupancy of each channel can be determined by measuring the energy of the signal at the output of each filter. The high complexity encountered by such approach is a major challenge as numerous RF components are required for the implementation. Wideband direct-conversion receivers (DCRs) ((a.k.a. homodyne, synchrodyne, or zero-intermediate frequency (IF) receiver) have become more and more popular due to their inherent advantages over superheterodyne receivers [9]. For instance, because the IF is zero, the image to the desired RF signal is the desired signal itself, which means DCRs do not face conventional image problems. Therefore, bulky, off-chip, front-end image-reject filters are unnecessary in DCRs. The direct-conversion architecture is highly attractive for integrated $\mathrm{RF}$ receivers as the reduction in off-chip components leads to higher levels of integration and lower costs [9]. However, DCRs (especially the low cost ones) suffer the RF impairments, for instance, I/Q imbalance and nonlinear distortion [9]. The nonlinearity causes signal compression and as a result introduces distortion and intermodulation. Consequently, they can cause false alarms in spectrum sensing when a vacant channel contains the distortion and is falsely interpreted as occupied by a primary user.

To reduce such an effect, the operating point of an amplifier must be set far from the compression point which in turn causes low power efficiency. This concept is called "power average back-off." Typically, additional back-off is also required to ensure that the transistor is not driven into saturation, where it becomes very nonlinear and creates substantial spectral 


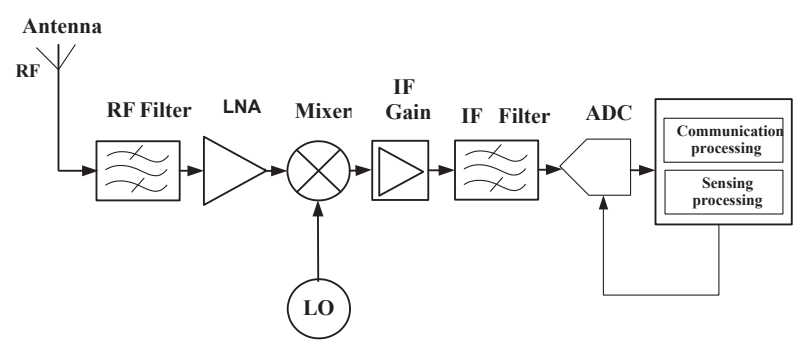

Fig. 1. Cognitive radio receiver structure.

regrowth. This behavior incurs a further loss of efficiency. Moreover, it is also not cost efficient since high cost transistors with much more power are needed for a sufficient back-off power. As a result, its implementation is costly. In addition, this power loss causes over-heating problem and shortens the battery life. The use of high cost transistors is also not feasible for mass-product devices, e.g., cognitive mobile phone. Therefore, a careful analysis of the nonlinearity of an LNA is necessary in order to operate without using such an expensive technique. To overcome this, many linearization techniques have been developed like feedback, feedforward, predistortion, etc. The linearization approach is not considered in this paper.

In this paper, we study the impact on the energy detection based spectrum sensing caused by a nonlinear low noise amplier (LNA) at the RF front-end of a wideband cognitive radio receiver. The nonlinearity with memory is modeled using a memory-polynomial model. We will see that threshold levels are needed to be set properly according to the different statistics along the whole frequency range.

\section{Signal AND System Models}

A simple structure of a cognitive radio receiver is illustrated in Figure 1. The radio frequency (RF) signal at frequency $f_{\mathrm{c}}$ is first captured by the RF antenna, passed through the RF filter and a low noise amplifier(LNA). It is then downconverted by a mixer and local oscillator (LO) to the intermediate frequency $f_{\mathrm{IF}}$, followed by an IF filter and subsequently by an analog-todigital converter (ADC) at RF. The received signal is further processed, i.e., communication and sensing processing in the digital domain. The scanned spectrum is divided into small sub-bands of interest and a decision about the occupation state of these sub-bands will be made depending on the analysis of the spectral density, i.e., either energy detection or other feature detection algorithms. The decision is made taking into account adjustable thresholds.

An LNA is the first amplifier in the RF receiver front-end; typically it is the first or second component after the antenna. It is designed to increase the power of the received signal which is usually very weak. LNAs are designed to add as little noise as possible, such that the signal to noise ratio (SNR) stays above the minimum required SNR of the receiver. A CR receiver equipped with a wideband radio front-end can observe multiple channels at a time, consequently it is more flexible as sensing multiple channels and can operate in parallel on the digital back-end, reducing the time to find a spectrum hole. However, wideband sensing receivers, which usually are DCRs, will operate in a range where the RF front-end components, such as the LNAs exhibit a nonlinear behavior introduced by the solid state transistors [10]. Therefore, intermodulation (IMD) and crossmodulation (XM) are generated [9]. The IMD terms that arise in wideband receivers can fall within the channel being downconverted. These IMD terms cannot be filtered out as in the narrowband approach since they overlap with the underlying primary user signals that need to be sensed. As a result, it is important to analyze the spectrum sensing degradation caused by wideband front-end nonlinearities. The baseband equivalent of the wideband signal after the nonlinear LNA can be then expressed as

$$
y_{b}[n]=G(x[n])+w_{a}[n],
$$

where the wideband signal can be written by $y_{w}[n]=$ $\mathcal{R}\left\{y_{b}[n] \exp \left(j 2 \pi f_{\mathrm{c}} t\right)\right\}, x[n]$ is the received baseband equivalent wideband signal and consists of $s[n]+w_{b}[n] . s[n]$ is the primary signal, whereas $w_{b}[n]$ and $w_{a}[n]$ are the additive thermal noise before and after the LNA, respectively. They are assumed to be white Gaussian process with zero mean and unit variance. $G(\cdot)$ specifies the nonlinearity model of the LNA. The memory effect is omitted in most of the existing literatures only due to simplicity [10]. However, the effect is always there in LNAs and really matters when signal statistics are tested for spectrum sensing. A nonlinear model with memory is considered in our study using a memory-polynomial model [11], which is the truncated version of the general Volterra series model [12]. The memory-polynomial consists of several delay taps and nonlinear static functions considering only diagonal terms in the Volterra kernels, thus, the number of the parameters is significantly reduced compared to the general Volterra series [12]. Thus, $G(x[n])$ in (1) can be represented with a memory polynomial model as

$$
G(x[n])=\sum_{q=0}^{Q} \sum_{k=1}^{K} a_{2 k-1, q}|x[n-q]|^{2(k-1)} . x[n-q],
$$

where $a$ is the model coefficients, $Q$ is the tap number, and $2 K-1$ is the order of the polynomial. This model considers only odd-order nonlinear terms due to bandpass nonlinear characteristics and the terms up to $2 n-Q$ that are considered in modeling because the even-order terms are usually outside of the operational bandwidth of the signal and can be easily filtered out [13]. An input signal, with a delay of up to $Q$ samples, is considered in this model. The scenario in a given subband of interest after downconversion to an intermediate frequency $f_{\mathrm{IF}}$ is considered. The signal at $f_{\mathrm{IF}}$ is written by

$$
y[n]=G(x[n]) \exp \left(j 2 \pi f_{\mathrm{IF}} t\right)+w[n],
$$

where the additive noise at $f_{\mathrm{IF}}$ is denoted by $w[n]$ with zero mean and variance $\sigma_{w}^{2}$. Note that as one of the other names of DCRs, i.e., zero-IF receiver, $f_{\mathrm{IF}}$ in (3) is equal to 
zero. That is DCRs demodulates the incoming radio signal using synchronous detection driven by a local oscillator whose frequency is identical to, or very close to the carrier frequency of the intended signal [9].

\section{Spectrum Sensing Methods And the Effect of NONLINEARITY WITH MEMORY}

We consider a simple Nyquist wideband sensing approach, i.e., the wideband received signal $y[n]$ is firstly sampled by a high sampling rate ADC, after which a serial to parallel conversion circuit is used to divide the sampled data into parallel data streams. A Fast Fourier transform (FFT) is used to convert the wideband signals to the frequency domain. The wideband spectrum $Y(f)$ is then divided into a series of subnarrowbands spectra $Y_{1}(f), \ldots, Y_{N_{\text {sub }}}(f)$, where $N_{\text {sub }}$ denotes the number of subnarrowbands. Each subnarrowband consists of $N_{\text {FFT }}$ frequency bins. The function of the spectrum sensing of cognitive radio devices is to detect whether primary users are absent or present in each subband spectra with two hypotheses, $\mathcal{H}_{0}$ and $\mathcal{H}_{1}$, respectively. A hypothesis test is formulated based on the type of detector. For instance, the energy detector [13] used in our study performs the following hypothesis test

$$
\begin{aligned}
& \mathcal{H}_{0}: E_{Y_{i}(f)}<\gamma(f), \\
& \mathcal{H}_{1}: E_{Y_{i}(f)}>\gamma(f),
\end{aligned}
$$

where $E_{Y_{i}(f)}$ is the energy in subband $i$ of the received signal $Y_{i}(f)$ and equals to $\sum_{f} Y_{i}(f) Y_{i}(f)^{*}$, and $\gamma(f)$ is the decision threshold that is set to maintain a minimum probability of false alarm $P_{\text {fa }}=\operatorname{Pr}\left\{E_{Y_{i}}>\gamma \mid \mathcal{H}_{0}\right\}$.

\section{A. Threshold Selection}

Firstly, without considering the nonlinearity distortion of the LNA, the threshold statistic is determined by the additive white Gaussian noise $w[n]$ after the FFT as

$$
W_{k}=\frac{1}{\sqrt{N}} \sum_{n=0}^{N-1} w[n] \exp \left(\frac{-j 2 \pi k n}{N}\right),
$$

where $k$ is the integers inbetween 0 and $N-1$. Since each value of $W_{k}$ is the summation of $N$ independent white Gaussian noise samples, $w[n]$, it follows that $W_{k}$ is an independent white Gaussian noise process. Therefore, the threshold level $\gamma(f)$ used in wideband sensing has the same central chi square distribution with $N$ degrees of freedom for the entire frequency range.

When the nonlinearity of the LNA is considered, the threshold level has to be carefully selected. For easier understanding, we expand $G(x[n])$ in (2) as

$$
\begin{aligned}
G(x[n])= & a_{1,0} x(n)+\ldots+a_{1, Q} x(n-Q), \\
+ & a_{3,0} x^{3}(n)+\ldots+a_{3, Q} x^{3}(n-Q), \\
& \ldots \\
+ & a_{2 K-1,0} x^{2 K-1}(n)+\ldots+a_{2 K-1, Q} x^{2 K-1}(n-Q) .
\end{aligned}
$$

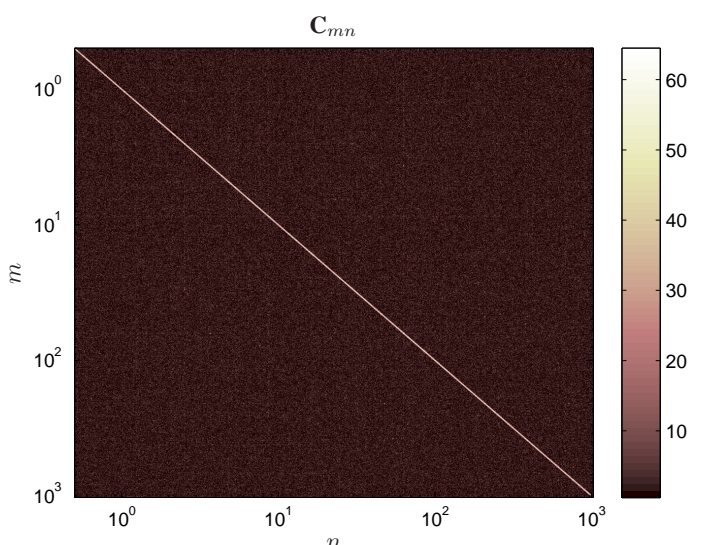

Fig. 2. Covariance matrix of the sequence of the noise amplitude after the nonlinear LNA.

When the primary signal is absent, the decision statistic is followed by $\sum_{n=1}^{N}\left|G\left(w_{b}[n]\right)+w_{a}[n]\right|^{2}$. First, we look at the probability density function (pdf) of $G\left(w_{b}[n]\right)+w_{a}[n]$, which is the convolution of the pdf of $G\left(w_{b}[n]\right)$ and the pdf of $w_{a}[n]$. The pdf of each component of $G\left(w_{b}[n]\right)$ can be analyzed using the function of random variable method [14], whereas the pdf of $w_{a}[n]$ is already known as a Gaussian distribution. We consider a random variable $Y$, which is related to another random variable $X$ as

$$
Y=g(X)=b X^{2 k-1}, k=1,2, \ldots, K,
$$

where $b$ is a constant. Due to the monotonic property of the function $g(X)$, the pdf of $Y$ can be expressed as

$$
\begin{aligned}
f_{Y}(y) & =f_{X}\left(g^{-1}(y)\right)\left|\frac{d g^{-1}(y)}{d y}\right|, \\
& =f_{X}\left(\left(\frac{y}{b}\right)^{\frac{1}{2 k-1}}\right) \frac{1}{\left|b^{\frac{1}{2 k-1}}\right|(2 k-1)} \mid y^{\frac{2-2 k}{2 k-1} \mid,} \\
& =\frac{1}{(2 k-1)\left|b^{\frac{1}{2 k-1}}\right| \sqrt{2 \pi}} \exp \left(-\frac{\left(\frac{y}{b} \frac{2}{2 k-1}\right)}{2}\right)\left|y^{\frac{2-2 k}{2 k-1}}\right|,
\end{aligned}
$$

where $X$ is a Gaussian distributed random variable with zero mean and unit variance. As we can see, when $k$ in (8) is equal to $1, Y$ is also a Gaussian distributed random variable. Otherwise, $Y$ becomes a non-Gaussian distributed random variable. The summation of correlated non-Gaussian and Gaussian random variables in (6) can be approximated using a multivariate Gaussian mixtures with the corresponding covariance matrices [15]. An example of the corresponding covariance matrices $\mathbf{C}_{m n}$ of the sequence of the noise amplitude after the nonlinear LNA is shown in Figure 2. Therefore, the threshold statistic is now determined by a correlated Gaussian sequence instead of a white Gaussian sequence as the input of the FFT.

\section{Simulation}

A Monte-Carlo simulation was run with $10^{4}$ realizations. All the other simulation parameters are listed in Table 1. The 
TABLE I

SIMULATION PARAMETERS

\begin{tabular}{c|c}
\hline Parameters & Values (Units) \\
\hline \hline Monte-Carlo run & $10^{4}$ (runs) \\
\hline The number of samples & 16384 (samples) \\
\hline Number of FFT points $\left(N_{f}\right)$ & 1024 (points) \\
\hline Nonlinearity model coefficients $(a)$ & {$[14.850-23.35 ; 033.830 ;-25.4207 .38]$} \\
\hline Target $P_{\mathrm{fa}}$ & 0.1 and 0.01 \\
\hline
\end{tabular}

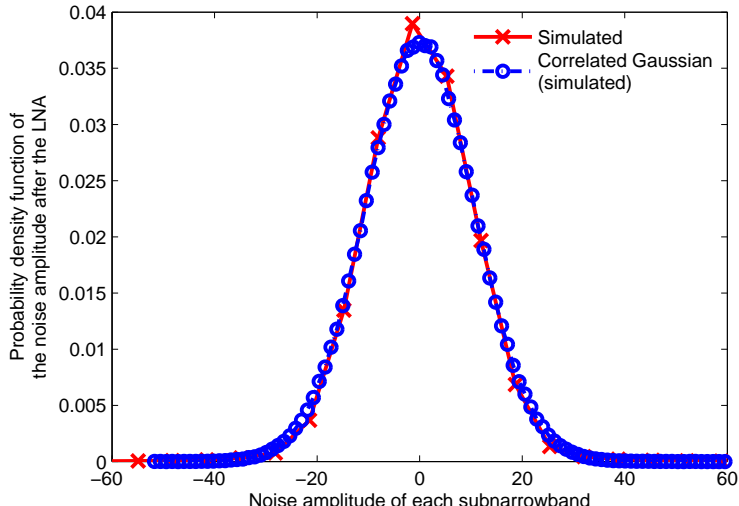

Fig. 3. Probability density function of the noise amplitude after the nonlinear LNA.

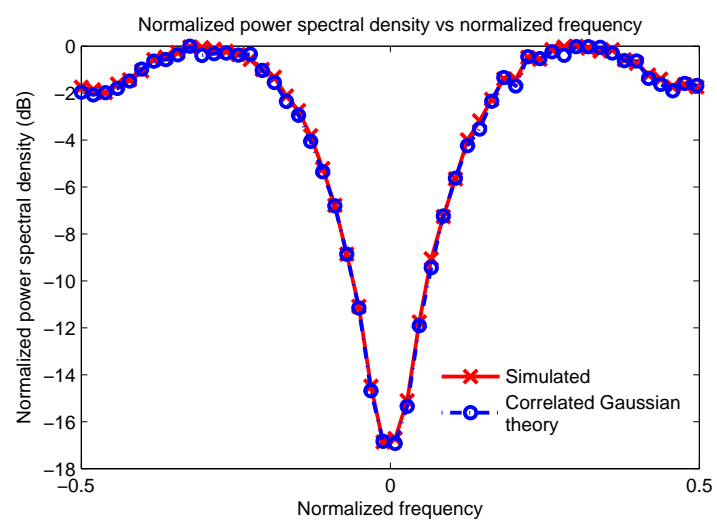

Fig. 4. Normalized power spectral density of each subnarrowband of the noise after the nonlinear LNA.

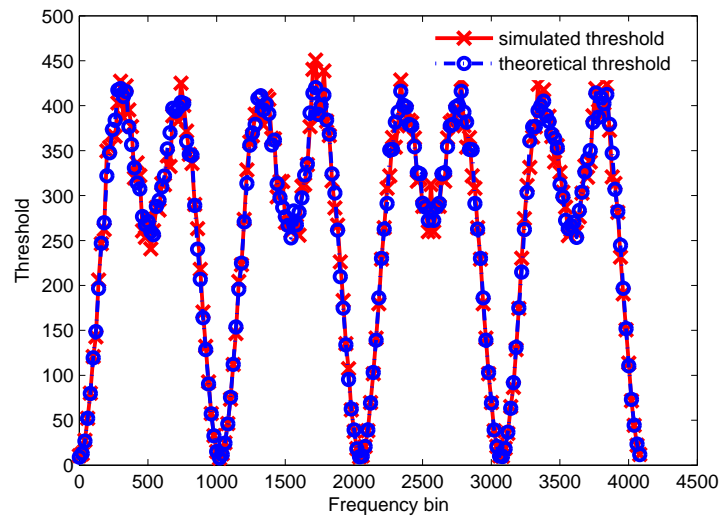

Fig. 5. The proper threshold level along the whole frequency.

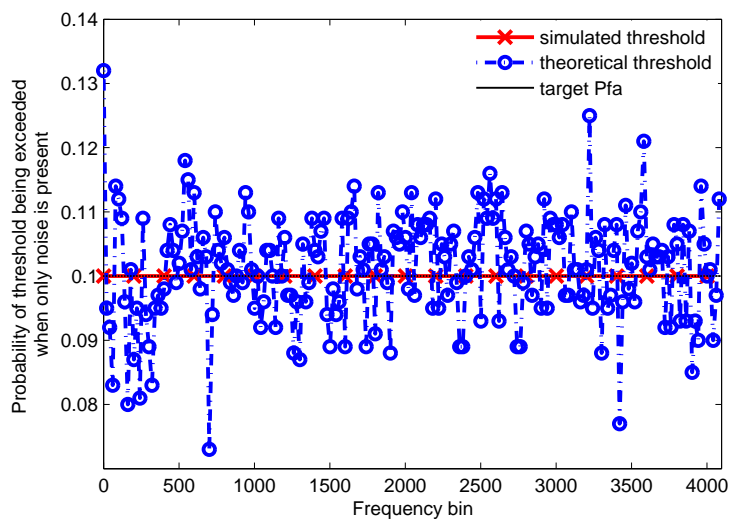

Fig. 6. The probability of threshold being exceeded when only noise is present for the target $P_{\mathrm{fa}}$ set to 0.1

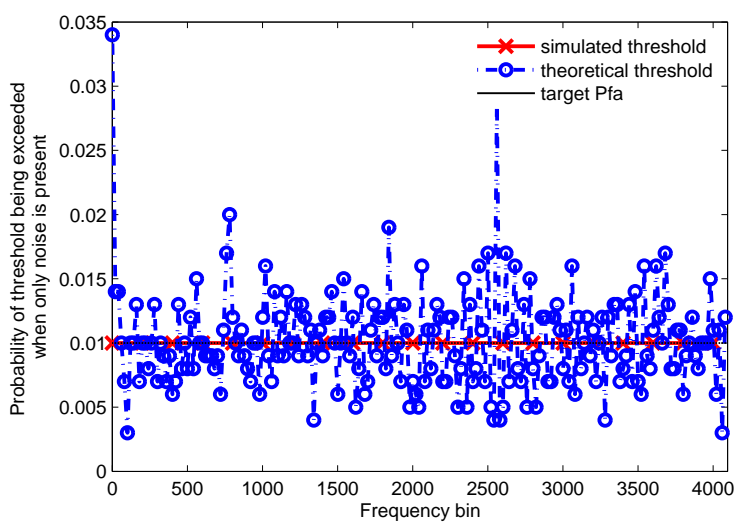

Fig. 7. The probability of threshold being exceeded when only noise is present for the target $P_{\mathrm{fa}}$ set to 0.01 .

memory polynomial coefficients $a$ are also given in Table 1 . The given coefficients means $Q=2$ and $K=3$, i.e., the third order and the fifth order IMD are considered. The evenorder terms are set to zero since they are usually outside of the operational bandwidth of the signal and can be easily filtered out as previously explained. The coefficients are chosen to be real in order to model amplitude-to-amplitude (AM-AM) distortion of the LNA. The pdfs of the noise amplitude after the nonlinear LNA are shown in Figure 3. We can see that the correlated Gaussian pdf fits the pdf of the nonlinear filtered noise better than the uncorrelated Gaussian pdf. The normalized power spectral density of each subnarrowband of the noise after the nonlinear LNA is shown in Figure 4. It shows that the noise after the FFT is better fitted by the correlated 
Gaussian random vector. The theoretical result curve fits very well the simulation one. To obtain the theoretical result, the affine transformation of Gaussian random variables is used to model the FFT [16]. And that, magnitude-squared complex Gaussians follow the exponential, where the mean level can be found using the affine transformation theory. Therefore, the threshold levels along the whole frequency can be properly set as the threshold statistic of each frequency bin has the exponential distribution as shown in Figure 5. As can be seen in Figure 6, the selected threshold level will give the probability of threshold being exceeded, when only noise is present, excellently matches the target $P_{\text {fa }}$ set to 0.1 . However, the reason of the fluctuation in the case of theoretical threshold is due to a finite number of samples used in the simulation. Therefore, the probability of threshold being exceeded, when only noise is present, in this case cannot exactly equal to the target $P_{\mathrm{fa}}$. Figure 7 confirms the validity of the result for the case of the target $P_{\text {fa }}$ set to 0.01 . To obtain those theoretical results, the affine transformation of Gaussian random variables was used to model the FFT [16]. And that, magnitude-squared complex Gaussians follow the exponential, where the mean level was found using the affine transformation theory.

\section{CONCLUSiON}

In this paper, we studied the impact of the nonlinear distortion caused by an LNA at the RF front-end of a wideband cognitive radio receiver. A memory-polynomial model was used to take into account the memory effect of this nonlinearity. The distribution of the noise introduced by the LNA is determined using a correlated Gaussian sequence. Based on the study, the proper threshold levels for different frequency bins can be set. This study can contribute to the topic of implementing low cost wideband cognitive radio devices.

\section{REFERENCES}

[1] J. Mitola, G. Maguire, "Cognitive radio: making software radios more personal, Personal Communications," IEEE Wireless Communications, vol. 6, no. 4, pp. 13-18, 1999.

[2] R. Tandra, S. M. Mishra, and A. Sahai, "What is a spectrum hole and what does it take to recognize one?," in Proc. IEEE, vol. 97, Mar. 2009.

[3] S. Shellhammer, S. Shankar, R. Tandra, and J. Tomcik, "Performance of power detector sensors of dtv signals in ieee 802.22 WRANs," in Proc. 1st ACM Int. Workshop Technol. Policy Access. Spectrum (TAPAS), Aug. 2006.

[4] H. Sun, D. Laurenson, and C.-X. Wang, "Computationally tractable model of energy detection performance over slow fading channels," IEEE Commun. Letters, vol. 14, no. 10, pp. 924926, Oct. 2010.

[5] Z. Tian and G. Giannakis, "A wavelet approach to wideband spectrum sensing for cognitive radios," in Proc. IEEE Cognitive Radio Oriented Wireless Networks and Commun., pp. 15, June 2006.

[6] B. Farhang-Boroujeny, "Filter bank spectrum sensing for cognitive radios," IEEE Trans. Signal Processing, vol. 56, no. 5, pp. 18011811, May 2008.

[7] Z. Tian and G. Giannakis, "Compressive sensing for wideband cognitive radios," in Proc. IEEE International Conference on Acoustics, Speech, and Signal Processing, pp. 13571360, April 2007.

[8] Z. Quan, S. Cui, A. H. Sayed, and H. V. Poor, "Wideband spectrum sensing in cognitive radio networks," in Proc. IEEE International Conference on Communications (ICC), pp. 901906, 2008.

[9] B. Razavi, "Design considerations for direct-conversion receivers," IEEE Trans. Circuits Syst. II, vol. 44, no. 6, pp. 428435, June 1997.
[10] M. Grimm, M. Allén, J. Marttila, M. Valkama, and R. Thomä, "Joint mitigation of nonlinear RF and baseband distortions in wideband directconversion receivers," IEEE Trans. on Microwave Theory and Techniques, vol. 62, pp. 166 - 182, Jan. 2014.

[11] L. Ding, G. T. Zhou, D. R. Morgan, Z. Ma, J. S. Kenney, J. Kim, and C. R. Giardina, "Memory polynomial predistorter based on the indirect learning architecture," in Proc. IEEE Global Telecomm. Conf., pp. 967971, Nov. 2002.

[12] M. Schetzen, The Volterra and Wiener Theories of Non-Linear Systems. New York: Wiley-Interscience, 1980.

[13] V. I. Kostylev, "Energy detection of a signal with random amplitude," in Proc. IEEE Int. Conf. on Commun. (ICC), pp. 16061610, May 2002.

[14] A. Papoulis, Probability, Random Variables and Stochastic Processes. $3^{\text {th }}$ Edition-McGraw-Hill, 1991.

[15] R. S. Blum , Y. Zhang , B. M. Sadler , R. J. Kozick, "On the Approximation of Correlated Non-Gaussian Noise Pdfs using Gaussian Mixture Models," Conf. Appl. Heavy Tailed Distributions Econom., Eng., Statist., Washington, DC, June 1999.

[16] H. Michiel, ed., "Affine transformation," Encyclopedia of Mathematics, Springer, ISBN 978-1-55608-010-4, 2001. 